\title{
Las construcciones escindidas como recurso pragmático para la expresión y configuración de la polémica en dos discursos sobre la izquierda
}

\section{Introducción}

Resulta cada vez más común en el análisis del discurso considerar la interfaz semántico-pragmática de ciertas estructuras sintácticas que ayudan a identificar algunos aspectos de naturaleza discursiva, como la modalida`d derivada del uso de oraciones adversativas (García Negroni 2009); el distanciamiento del locutor con respecto a su enunciado, ya sea para otorgar efectos de objetividad o para suspender el compromiso enunciativo, mediante la voz pasiva (Montolío 2001) o el discurso referido (Nascimento 2015); la construcción de cohesión y coherencia con las categorías de foco y fondo (Bublitz 2011); los indicadores de cambio temático en discursos específicos (Cortés e Hidalgo 2015), o la determinación del punto de vista por medio del fondo y la figura (grounding) (Ehrlich 1990). En esta línea, buscamos contribuir en las disciplinas afines al análisis del discurso con el estudio de las construcciones escindidas como un recurso que puede ayudar a orientar la confrontación de puntos de vista y la intención polémica del locutor entre las distintas opiniones que se confrontan y resuenan en su discurso.

El presente trabajo se inscribe dentro de los estudios de discurso político mexicano, al analizar dos discursos antagónicos sobre la izquierda, el anarquista y el socialdemócrata. Particularmente se enfoca en el tratamiento polémico de tres pares de objetos discursivos que son relevantes para ambas corrientes: izquierda/no izquierda, revolución/reforma y anticapitalismo/antineoliberalismo, los cuales mantienen entre sí relaciones complejas, sobre todo de oposición.

El análisis que se describe profundiza en el papel de las estructuras escindidas para configurar y expresar la dimensión polémica (Amossy 2017) desde una perspectiva polifónica (Bajtín 1979, Ducrot 1984). Adoptar esta perspectiva teórica nos permite considerar las estructuras escindidas como una marca de heterogeneidad enunciativa (Authier 1995); es decir, como formas que inscriben

Nélida Murguía, Universidad de Buenos Aires, Ciudad Autónoma de Buenos Aires, Argentina

Ә Open Access. (C 2019 Nélida Murguía, published by De Gruyter. (cc) BY-NC-ND This work is licensed under a Creative Commons Attribution-NonCommercial-NoDerivatives 4.0 International License.

https://doi.org/10.1515/9783110605679-011 
en el discurso propio la voz de un otro social, en este caso antagonista, con el fin contrastar puntos de vista.

La organización del capítulo es como sigue: Primero precisamos la conformación del corpus y describimos la tipología de las construcciones escindidas a partir del material recogido; posteriormente, detallamos el potencial polémico de las escindidas por medio de la focalización y el contraste pragmático, y, finalmente, abordamos el análisis de diversos ejemplos del discurso de izquierda elegido para ilustrar la dimensión polémica y su construcción de sentido.

\section{Conformación del corpus}

El corpus proviene de una serie de entrevistas realizadas a 15 personalidades de la izquierda mexicana (Centro de Documentación y Difusión de Filosofía Crítica 2013). De este corpus se analizan dos discursos, elegidos porque representan dos posturas controversiales y los entrevistados participan en movimientos $\mathrm{u}$ organizaciones en donde ejercen un papel de influencia ideológica. La postura anarquista (A) está representada por Alfredo Velarde, quien se identifica como anarcocomunista y participa en diversas agrupaciones dentro y fuera de la Universidad Nacional Autónoma de México (UNAM). En el polo contrario, la orientación socialdemócrata (S) está representada por Héctor Díaz-Polanco, quien participa activamente en el Movimiento Regeneración Nacional (Morena), asesorando y formando política e intelectualmente a otros militantes. Ambos son académicos de la UNAM. Cabe precisar que las entrevistas, cuya duración es de aproximadamente una hora, fueron realizadas en diferentes momentos y que los entrevistados nunca interactuaron cara a cara. El análisis se lleva a cabo a partir de una transcripción simple de los videos de las entrevistas y sin considerar fenómenos paratextuales.

El corpus resultante es de 71 construcciones escindidas de tres tipos, los cuales se describen a continuación. La clasificación que se presenta se basa en la taxonomía propuesta en De Cesare (2014), lo que nos permite dar cuenta de otras estructuras emparentadas, además de las tradicionalmente consideradas como escindidas en la literatura (escindidas, pseudo-escindidas y pseudoescindidas inversas). 


\section{Construcciones escindidas analizadas}

\subsection{Tipo I: “Escindidas"}

En esta categoría incluimos las estructuras consideradas tradicionalmente en español como escindidas, pseudo-escindidas y pseudo-escindidas inversas. Las escindidas del Tipo I reúnen todas las características que se han reconocido para estas construcciones en la literatura: pueden convertirse a una sola oración semánticamente equivalente, son especificativas y están compuestas por tres elementos que pueden presentarse en distinto orden: constituyente escindido + cópula + oración relativa. En este grupo, la relativa es libre o semilibre (es decir, sin antecedente expreso), y está introducida por un pronombre o adverbio relativo: que, quien, art. + que, o bien, cuando, donde, como, cuanto (NGLE 2010, $\S 44.4 .1,44.4 .2$.); por ejemplo, en (1):

\section{(1) quien asumió el cargo de Rector de la UNAM fue Enrique Graue. ${ }^{1}$}

En este mismo rubro hemos considerado las oraciones introducidas por artículo + que (las cuales son relativas semilibres), debido a que forman parte de las que suelen considerarse clásicamente en español como escindidas o pseudoescindidas, a diferencia del tipo II, que son más atípicas en la literatura y tienen más rasgos que las diferencian. ${ }^{2}$

\subsection{Tipo II: “Escindidas ampliadas”}

Este tipo de construcciones tienen una relativa con antecedente, el cual puede ser un sustantivo (genérico o no genérico), un numeral o un cuantificador (Collins 1991). Este antecedente en ocasiones hace cuestionable que se correspondan con una estructura indivisa con el mismo significado.

(2) Lo único que publicaron en las noticias fueron hechos sin importancia

\footnotetext{
1 Todos los ejemplos de esta sección fueron construidos para ilustrar cada tipo de construcción. Los datos del corpus se presentan a partir de $\S 4$.

2 De Cesare, Garassino, Agar Marco y Baranzini (2014) las reúnen junto con las que aquí hemos clasificado como tipo II, pues consideran que la oración escindida es una oración encabezada por una frase nominal (realizada en forma de pronombre) con subordinada de relativo, al igual que las del tipo II.
} 
Nótese que la conversión debe ser diferente para conservar el significado: Publicaron en las noticias únicamente* hechos sin importancia. En inglés y alemán, este tipo de estructuras han sido llamadas focusing copular sentences with generalized nouns (Erdmann 1990). A diferencia de las escindidas típicas, pueden ser interpretadas como monoclausales, predicativas, que incluyen una frase nominal anafórica, en vez de ser biclausales y especificativas (Calude 2009). Sin embargo, el principal criterio para incluirlas en este estudio (siguiendo a De Cesare 2014) es que, tal como las escindidas típicas, son copulativas, contienen una oración de relativo especificativa y generan un foco contrastivo; es decir, cumplen la misma función pragmática y discursiva. Como en nuestro caso, algunos autores incluyen las encabezadas por sustantivos semánticamente no genéricos y las subclasifican de acuerdo con este rasgo (De Cesare 2014, Agar Marco 2014).

Dentro de esta categoría incluimos también estructuras en donde se repite una parte de la frase nominal del constituyente escindido en la oración escindida, como en (3):

(3) El problema ecológico es el problema donde debemos poner mayor atención en la actualidad

\subsection{Tipo III: “Escindidas reducidas o no verbales"}

Este tipo de estructuras ha sido estudiada en diferentes lenguas (España 1996, Atayan y Wienen 2014). Se trata de construcciones copulativas especificativas donde el verbo ser aparece en tercera persona del singular y se une a una oración sustantiva introducida por el pronombre que: “es que”. Una de sus funciones es explicar o introducir una conclusión, pero también pueden generar un contraste, por su carácter especificativo. En estas construcciones, la cópula no une a la oración sustantiva (el constituyente escindido) con una oración escindida (pues el elemento que cumple esta función no es verbal: no hay oración de relativo o una frase que la contenga), sino a una frase nominal simple, como en $a$ ), o incluso puede estar ausente, como en $b$ ).

a) Introducidas por una frase nominal simple + es que. La frase nominal puede ser evaluativa ("lo bueno") o un sustantivo operador. Algunos autores (como Smead 1994) las han llamado seudoescindidas no verbales.

(4) lo verdaderamente acuciante de las próximas elecciones es que hay mucha corrupción 
b) Iniciadas por es que. Fernández Leborans (1992) las denomina clefts reducidas; mientras que Atayan y Wienen (2014), escindidas inferenciales. Son fórmulas gramaticalizadas e incluso marcadores discursivos empleados para introducir un argumento explicativo de una conclusión explícita o implícita (España 1996). Crean un contraste pragmático, ya que funcionan para anular una creencia hipotética o real que circula en el universo discursivo.

(5) es que los intereses de la izquierda no deberían limitarse a la clase obrera, sino a todo aquel vulnerado en sus derechos

\subsection{Frecuencia de construcciones escindidas}

El corpus reunido se compone de 71 estructuras escindidas de los tres tipos descritos, encontradas en 19,500 palabras. ${ }^{3}$ Cabe mencionar que se observó una tendencia a que los constituyentes escindidos aparezcan en la posición final de la construcción (50; es decir, $71 \%$ ) y a que la oración escindida sea una relativa libre o semilibre (36, es decir, $51 \%$ ). Por el contrario, las estructuras que menos se utilizan son las del tipo III y la posición en la que menos aparece el constituyente escindido es la media, apenas 3\%; esta posición correspondería en inglés a la de la IT-cleft; es decir, a la prototípica. Estas tendencias se sistematizan en la Tabla 1, a partir de los 38 casos del discurso anarquista y los 33 casos del discurso socialdemócrata.

\section{Contraste pragmático y dimensión polémica}

Es posible entablar una relación entre las construcciones escindidas y la dimensión polémica a partir de la función discursiva de estas estructuras; es

3 Desafortunadamente esta frecuencia no es comparable con otras, debido a que hay pocos estudios que presenten datos similares en corpus similares. Por ello, no es posible afirmar o negar que exista una relación entre el empleo de estas construcciones y el género discursivo, en este caso político, o el medio, es decir, oral. Los estudios en lo que encontramos frecuencias normalizadas los datos no son comparables, pues, por ejemplo, en el caso de De Cesare, Garasino y otros (2014, tabla 11) se contabilizan sólo con constituyente medio y final. O bien, hay estudios que muestran frecuencias pero que no toman en cuenta el tipo II y III que aquí contabilizamos. Sin duda es un trabajo interesante, que por ahora quedará pendiente, para poder afirmar con seguridad la relación entre el género o el medio y el empleo de estas estructuras contrastivas. 
Tabla 1: Frecuencia de construcciones escindidas por tipo y posición del constituyente escindido.

\begin{tabular}{lrrrr}
\hline Tipo de escindida & I & II & III & Subtotal \\
\hline Posición del constituyente escindido & & & & \\
\hline Inicial & 14 & 5 & 0 & 19 \\
Medio & 0 & 1 & 1 & 2 \\
Final & 22 & 15 & 13 & 50 \\
\hline Subtotal & 36 & 21 & 14 & Total: 71 \\
\hline
\end{tabular}

decir, gracias a la focalización contrastiva que conecta, opone y selecciona una perspectiva frente a otra $\mathrm{u}$ otras, ya sea efectivamente expresadas en el propio discurso, o bien, que remiten a voces sociales que circulan en la producción global de discursos sobre determinado asunto.

De acuerdo con Garassino (2014) y Gutiérrez Bravo (2008), la función básica de las escindidas es resaltar el constituyente escindido para conectarlo con otras partes del discurso e identificarlo como un foco contrastivo con respecto a ellas. Podemos definir la focalización contrastiva como un procedimiento mediante el cual se hace presente una serie de posibilidades entre las que el enunciador destaca y elige una, eliminando a las otras. Es decir, despeja una incógnita informativa, al decantar información entre alternativas (Kiss 1998, Bosque y Gutiérrez-Rexach 2009, Van der Wal 2015). En este proceso, el enunciador enfatiza un elemento como el único y elimina al resto por medio de una operación pragmática vinculada con el carácter especificativo de las escindidas. Por supuesto, la lengua tiene diversos medios para efectuar este proceso aparte de estas construcciones, tales como la corrección, el orden de palabras, la marcación prosódica o el uso de operadores de foco, como único, solo, más bien, entre otros.

Huber (2006) llama a este proceso de decantación de información contraste pragmático y puntualiza que no resulta únicamente del realce del constituyente escindido, sino de una oposición que se da entre dicho constituyente y otras alternativas presentadas en el discurso. Para ejemplificar el contraste pragmático en las escindidas, véase la siguiente secuencia tomada de la traducción de un texto literario (La señora Dalloway, de Virginia Woolf):

(6) el vestíbulo se llenaría de caballeros esperando [...], mientras las señoras se quitaban las capas en la habitación del pasillo; ahí es donde las ayudaba la señora Barnet [...] (Woolf 2013, s/p). 
La función discursiva de la construcción escindida lleva a inferir que, al menos, hay otra alternativa posible además de la habitación del pasillo (ahí) como espacio en donde la señora Barnet podría ayudar a las damas a quitarse las capas; sin embargo, la oposición hace que ésta resulte eliminada. Así, el contraste pragmático en (6) es entre el constituyente escindido ahí (que sustituye a la habitación del pasillo) y el vestíbulo. Nótese que aquello no ocurriría si (6) hubiese sido expresado sintácticamente de otro modo: el vestíbulo se llenaría de caballeros esperando, mientras las señoras se quitaban las capas en la habitación del pasillo, donde las ayudaba la señora Barnet. Dicho así, no hay una especificación del espacio donde ocurre la acción, sino simplemente una descripción. El contraste entre ambos espacios (el vestíbulo y la habitación) funciona sobre todo para ordenar de manera espacial las acciones de los personajes y, probablemente, subrayar un mayor interés por el lugar femenino y sus características, lo cual en sí mismo no es polémico, pese a que podría serlo si se ponen en discusión cuestionamientos de género.

Lo anterior significa que no podemos asociar a priori el contraste pragmático con la intención polémica, pues no siempre marca dos o más puntos de vista opuestos. El carácter polémico lo otorga también el contexto donde se utilizan las construcciones escindidas, el género en el que aparecen, el registro que se emplea e incluso el tema del que se trata.

En el caso del discurso político, la dimensión polémica es un componente central, pues la discusión sobre asuntos que afectan a la sociedad, dentro de una sociedad democrática, implica la interacción de distintos puntos de vista que se relacionan de manera compleja, siendo la oposición una posibilidad. Las construcciones escindidas son sólo una de las marcas lingüísticas que podrían expresarla.

De acuerdo con Ruth Amossy (2017), la polémica es una confrontación de opiniones, cuya instauración y reproducción tiene un componente discursivo en el que se actualizan y hacen presentes dos discursos; socialmente, funciona para gestionar el conflicto, lo cual no significa que haya acuerdos resultantes ni que su modelo sea el diálogo. Más bien, contribuye a reforzar la identidad de quienes se identifican con determinada opinión y ello sienta las bases de una lucha en común que puede desembocar en acciones, incluso en un movimiento social.

La polémica ejerce un modo de razonamiento que posee tres procedimientos característicos, los cuales se identifican en el análisis de los ejemplos presentados el este capítulo: a) la dicotomización, al hacer excluyentes dos posiciones y eliminar las intermedias; $b$ ) la polarización, al establecer campos enemigos y plantear un "nosotros" frente a un "ellos", y c) el descrédito del otro (Amossy 2017). 
Para poder comprender la afirmación de que la polémica "hace presentes dos discursos" y el hecho de que pueda ocurrir sin que exista necesariamente la interacción cara a cara (como ocurre con las entrevistas de nuestro corpus), es necesario partir de una perspectiva polifónica del lenguaje (Bajtín 1979, Ducrot 1984, Authier 1995). Esta perspectiva cuestiona la existencia de un enunciador único y de un mensaje monódico; por el contrario, afirma que las enunciaciones están formadas por distintas voces, aunque su locutor efectivo sea uno solo. Es decir, considera al discurso como un abanico de voces distintas con respecto a las cuales el sujeto se posiciona para construir y expresar su punto de vista, a la vez que acepta, cuestiona, refuta, se identifica, se opone o silencia otras voces. Se trata de la representación en discurso del discurso del otro (Bajtín 1979).

En ese sentido, Amossy (2017) precisa que la polémica, aunque se exprese entre individuos concretos, no trata sobre disensos individuales, sino que se hace con una apelación al otro social y en relación con una polémica pública definida por la circulación global de discursos.

Siguiendo a García Negroni (2009), estas voces con las que interactúa el autor del enunciado se manifiestan a través de múltiples fenómenos lingüísticos, como la negación, el discurso referido, la ironía, la presuposición y la focalización, entre otros, algunos de los cuales son de naturaleza sintáctico-pragmática.

En nuestro caso, trabajamos con los discursos de dos sujetos que provienen de una memoria discursiva (Charaudeau 2001) ligada a posturas históricamente encontradas (la socialdemocracia y el anarquismo) pero con un objetivo común: la transformación hacia una sociedad más igualitaria (Bobbio 1995), cuyas voces se insertan y navegan en una circulación de discursos que ponen en escena en su propio decir. De esta manera, discuten sobre temas similares o, incluso, el mismo tema, pero desde posturas disímiles, y para hacerlo se posicionan con respecto al otro social.

La focalización contrastiva de las construcciones escindidas resulta ser particularmente productiva para mostrar cómo prevalece un único sentido, como ocurre en el discurso polémico (Garand 1989), y observar la puesta en escena de las diferentes voces. Veamos un ejemplo del discurso A, relacionado con uno de los temas polémicos que trabajamos en este capítulo, el de reforma o revolución.

(7) lo que es violencia en el capitalismo mexicano de las crisis recurrentes es la política neoliberal que ha fabricado millones de pobres. (A308)

El ejemplo (7) no sólo significa que "la política neoliberal que ha fabricado millones de pobres” (el foco contrastivo) constituya un acto de violencia, 
sino que, entre todas las cosas que podrían considerarse violencia, sólo esa lo es. El contraste pragmático permite inferir entonces que hay otras alternativas que, según otras voces, son consideradas como instancias de violencia, pero de las cuales el locutor se distancia y excluye del sentido que él otorga al concepto. Para reconstruir los puntos de vista con los que polemiza, podemos conectar con otras partes del discurso, donde se hace mención explícita del tema. Asimismo, gracias al carácter público de los disensos, podemos reforzarlo al evocar el debate sobre la violencia en el ámbito de la izquierda. En efecto, dentro del ámbito de la izquierda hay voces a favor de un cambio pacífico (la corriente reformista) y voces que piensan necesaria una transformación violenta (la corriente revolucionaria), (Bobbio 1995). Dentro de la propia entrevista encontramos esta oposición explicitada en fragmentos como los siguientes:

(8) hay una izquierda socialdemócrata que emprende una crítica de la violencia revolucionaria en términos metafísicos. (A303)

(9) hay una diferencia entre la violencia revolucionaria y la violencia reaccionaria, entre la violencia practicada por los oprimidos y por los opresores. (A206)

(10) ¿Que no tiene derecho el crisol de las organizaciones sociales civiles y populares ante las repetidas agresiones del principio de autoridad gubernamental, a ejercer el elemental derecho a la autodefensa? que es precisamente lo que me ha llevado recientemente a coincidir mucho con los compas de la CRAC [...] (A312)

De esta manera, pragmáticamente, (7) no sólo significa que la política neoliberal es violencia, sino que la violencia revolucionaria, la de los oprimidos y la autodefensa (alternativas eliminadas) no lo son realmente. Además, se genera una polémica con la postura de la izquierda socialdemócrata que sustenta, de acuerdo con el enunciador y con el debate público, lo contrario. Por otro lado, con respecto a los diferentes modos de razonamiento de la polémica, encontramos una dicotomización entre violencia y no violencia, y una polarización entre un nosotros que ejecuta un derecho; un ellos que agrede (el gobierno, la política neoliberal, los reaccionarios), y un tercero, más cercano al ellos, que es la izquierda socialdemócrata que critica un derecho legítimo sin comprender ("en términos metafísicos") el fenómeno. 


\section{Discurso polémico de izquierda}

A manera de marco de lectura para las secuencias analizadas que presentamos en este capítulo, describimos brevemente los resultados de un análisis discursivo en el que se estudiaron las representaciones sociales sobre la izquierda en tres movimientos mexicanos (anarquismo, socialdemocracia y movimiento urbano popular), ${ }^{4}$ con especial énfasis en los recursos de focalización y modalidad como marcas de posicionamiento con respecto a los objetos discursivos que se identificaron en los propios materiales (Murguía 2016). La teoría que sustentó este trabajo fue la de las representaciones sociales (RS) desde una perspectiva estructural (Abric 2004), la cual permite hacer una especie de captura de la RS en determinado espacio/tiempo para dar cuenta de su contenido y organización, donde los elementos constitutivos de la misma están jerarquizados en un sistema central y uno periférico.

En este acercamiento a las RS (Abric 2004, Bingono 2011) se postula la existencia de un núcleo estable, de carácter normativo, que genera unidad, significado y coherencia a toda la RS. El núcleo otorga significación y organiza al resto de los elementos a través de los lazos que establece con ellos. El sistema periférico, por su parte, es más flexible, da concreción al elemento central y puede adaptarse a las circunstancias propias del contexto; tiene elementos más cercanos y otros más alejados del núcleo. Además, tal como lo proponemos en Murguía (2016), pueden identificarse al menos dos polos: uno negativo y otro positivo, determinados fundamentalmente por la modalidad evaluativa expresada en el discurso. A partir de lo anterior, podemos afirmar que diferentes RS que pertenecen al campo abstracto de la izquierda, como en el caso del anarquismo y la socialdemocracia, pueden contener los mismos elementos; sin embargo, se diferencian por su jerarquización y valoración, lo cual otorga una significación y coherencia particular a cada RS.

Por otra parte, Moscovici (1988), el fundador de esta teoría, postula la existencia de tres tipos de RS: las hegemónicas, las emancipadas y, como en el caso que aquí se discute, las polémicas. Estas últimas emergen entre grupos que atraviesan situaciones de conflicto en relación con objetos socialmente relevantes y expresan puntos de vista divergentes. Como se observa en la Figura 1 y 2, las RS del anarquismo y la socialdemocracia son polémicas, pues en ellas no sólo es importante definir qué compone y determina a la izquierda, sino qué no pertenece a ella.

4 Se omite lo correspondiente a este movimiento, por no interesar en el presente capítulo. 


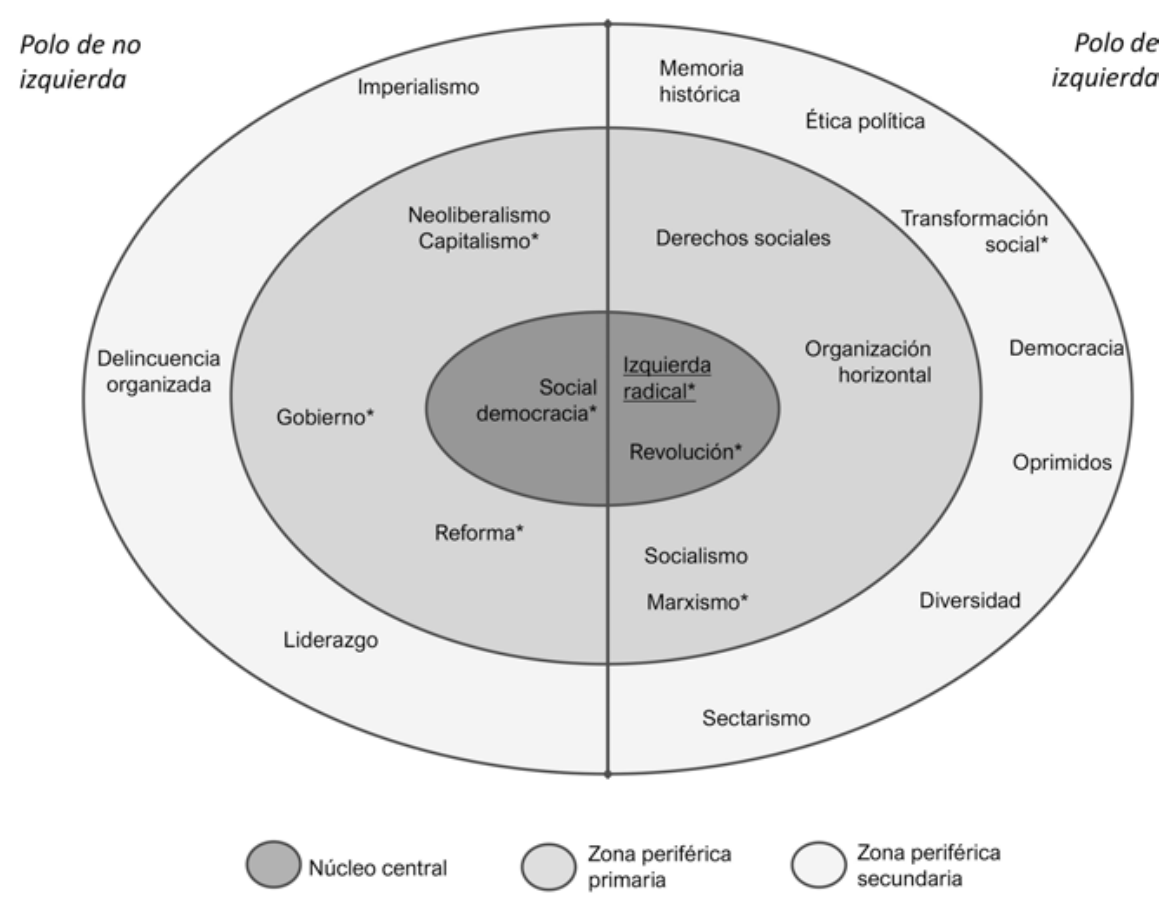

Figura 1: Representación social de la izquierda en el discurso anarquista.

Al observar las Figuras 1 y 2, resulta evidente que tanto la RS del anarquista como la del socialdemócrata son, valga el término, representaciones escindidas; es decir, están configuradas sobre la distribución de dos polos igualmente importantes. En uno de ellos se encuentra la izquierda y elementos asociados a ella, mientras que en el otro se ubica la no izquierda y aquello a lo que se opone. En otras palabras, se trata de una representación de naturaleza polémica. Para acentuar lo anterior, resulta también evidente que las fronteras de cada uno tienen distinto alcance; mientras que las de la socialdemocracia son ligeramente laxas; las del anarquista son estrictas, a tal punto que ningún tipo de socialdemocracia entra en su RS sobre la izquierda.

Haciendo una paráfrasis de la RS sobre la izquierda en el discurso A ilustrado en la Figura 1, podemos decir que tiene su núcleo central en la perspectiva radical revolucionaria que exige la organización horizontal, hecha desde la autonomía, y la búsqueda de derechos sociales. Además, se sitúa tajantemente en contra de las estructuras de poder y dominación, cuyo mejor representante es la socialdemocracia, a la que considera parte del sistema capitalista sostenido por el Estado y la derecha. Por otro lado, la 


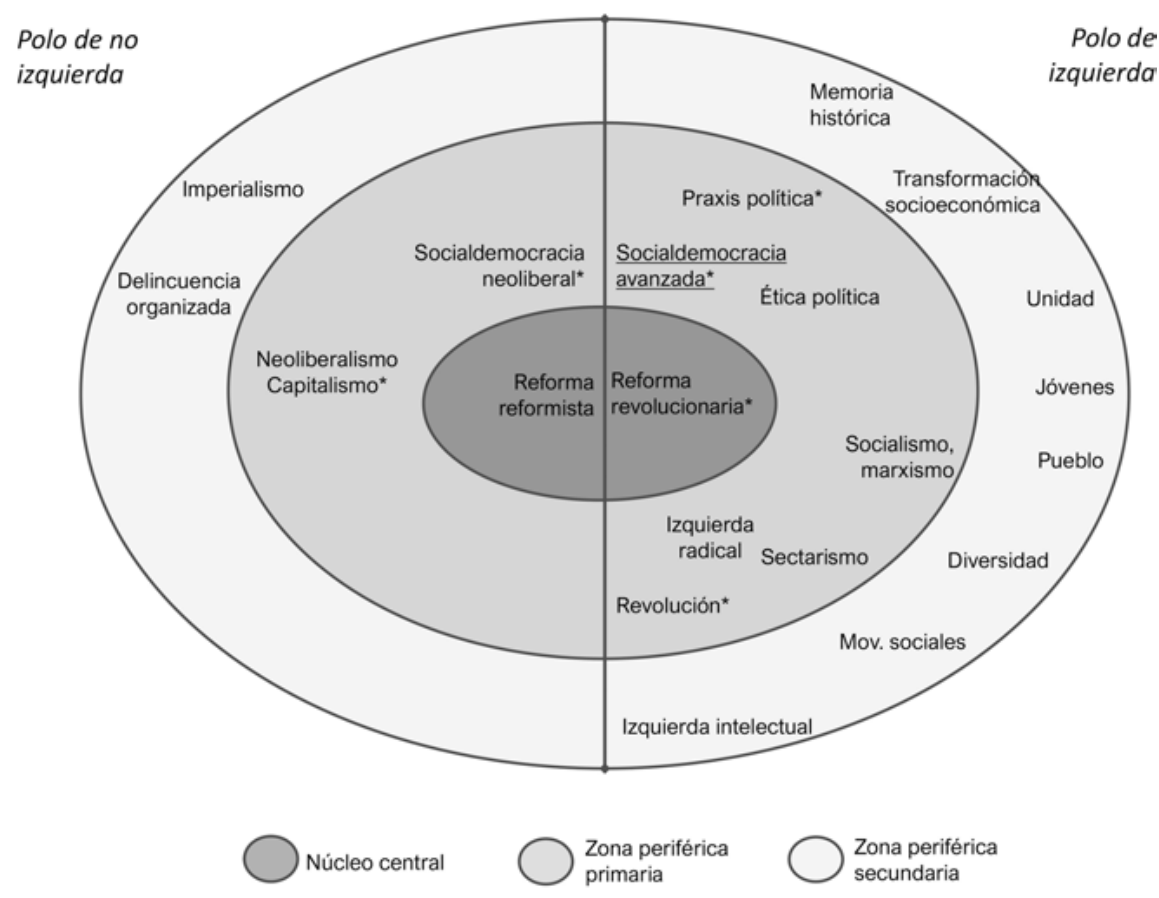

Figura 2: Representación social de la izquierda en el discurso socialdemócrata.

representación del socialdemócrata tiene su núcleo central en la necesidad de emplear el poder (reforma), a través de una organización política tradicional (partido), como expresión de la praxis política, y con objetivos transformadores revolucionarios. Se opone de manera central a la reforma de la socialdemocracia neoliberal.

Para los fines de este capítulo, nos enfocaremos en aquellos elementos constitutivos en cuya puesta en discurso se emplearon construcciones escindidas (están marcados con un asterisco en las representaciones gráficas de las Figuras 1 y 2). Como hemos mencionado, el contraste pragmático que generan estas estructuras sintácticas lleva a inferir que, adicionalmente al punto de vista explícito en la secuencia, hay al menos una alternativa eliminada o desacreditada dentro de la perspectiva del enunciador. Es decir, su potencial polémico aporta al significado expresado la existencia de voces divergentes anuladas.

De hecho, como podemos observar en la Figura 3, se trata de elementos que se componen de pares contrarios que, en este caso, indican los límites entre lo que puede ser considerado de izquierda y lo que no, de acuerdo con 


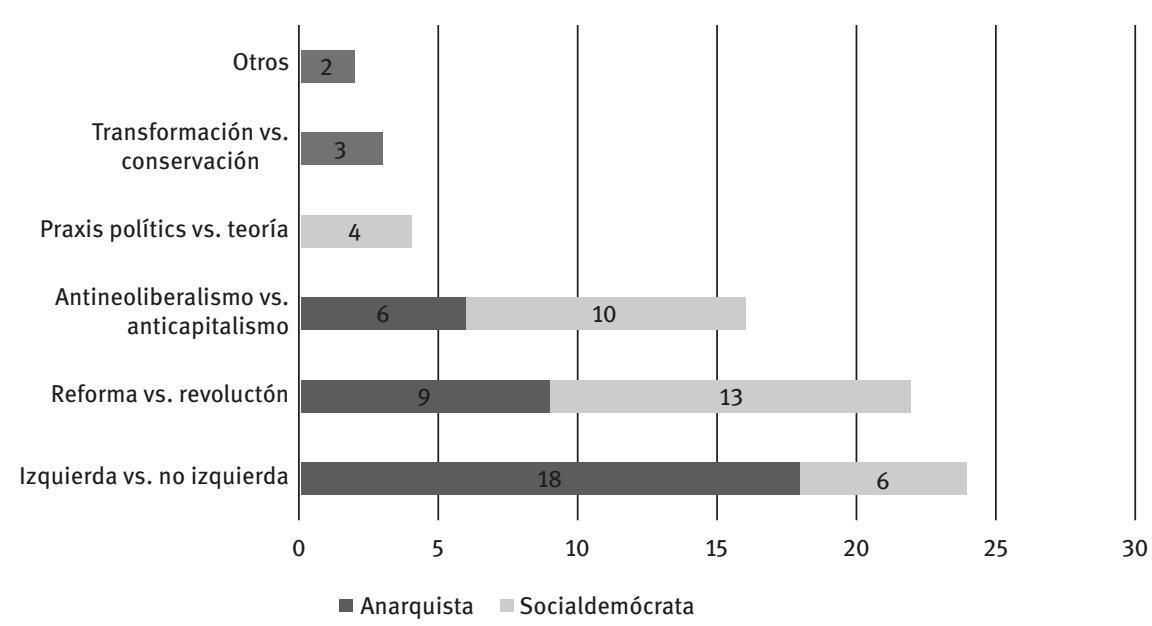

Figura 3: Frecuencia de uso de oraciones escindidas por tema agrupado.

ciertas características y fines. En la Figura 3 se grafica la frecuencia de oraciones escindidas por cada par en los dos discursos.

Los polos centrales se asientan entre lo que para cada uno es la izquierda y la no izquierda. Por supuesto, no se trata de una oposición abstracta, sino que se sostiene sobre la base de otras dicotomías que, en este caso, resultaron ser la de reforma vs. revolución y anticapitalismo vs. antineoliberalismo, las cuales están relacionadas.

Aparecieron en nuestros datos sobre las construcciones escindidas otras dicotomías y oposiciones que interesaron sólo a alguno de los dos: en el discurso A, transformación vs. conservación, así como la oposición al Gobierno y el Estado, y al marxismo; en el discurso S, la dicotomía entre teoría y praxis. No resulta sorprendente, dado que, mientras el anarquista está contra toda estructura de poder y por un cambio radical, el socialdemócrata se vale de dichas estructuras y considera que es la única manera de conseguir mejoras, a la vez que califica su postura como una de carácter realista, que incide en la práctica, más allá de las teorías que se hagan al respecto.

A continuación, revisaremos algunas de las secuencias consignadas, con el fin de ejemplificar la forma en la que se van construyendo las oposiciones y la polémica, expresadas en las construcciones escindidas. Con el objetivo de profundizar en los elementos que ambos comparten, nos enfocaremos en: izquierda $v s$. no izquierda, reforma $v s$. revolución y anticapitalismo $v s$. antineoliberalismo. 


\subsection{Fronteras de la izquierda}

En la Figura 3, puede visualizarse que el tema más frecuente es el de izquierda y no izquierda (con 24). En la tabla siguiente se presentan desglosados los datos que lo componen.

Conviene precisar las diferentes formas con que ellos nombran lo que identifican como izquierda y, por el contrario, como no izquierda. La etiqueta de no izquierda se refiere específicamente a las tendencias o grupos que se asumen públicamente como izquierdistas, pero que en opinión del enunciador no lo son. En el discurso A, son llamadas como autodenominadas izquierdas, centroizquierda progresista, izquierda partidocrática, centro dizqueprogresista e izquierda legal; mientras que la sí izquierda es nominada como verdadera, genuina, revolucionaria, radical y “que no se resiste a serlo”. En el caso del discurso S, la no izquierda es mencionada como socialdemocracia neoliberal, reformistas y reformismo socialdemócrata; en cambio, la sí izquierda es socialdemocracia avanzada o socialdemocracia "con diversos grados de radicalidad".

Al observar detenidamente las frecuencias de la Tabla 2 dos hechos saltan a la vista: el primero es que esta distinción resulta más relevante para la corriente anarquista; si volvemos a la Figura 1 advertiremos que el núcleo central de su representación está configurado de ese modo (la dicotomía central es entre izquierda radical y socialdemocracia - la cual no considera de izquierda-). Es decir, que la polémica principal que sostiene su representación es polarizar a sujetos y actores políticos. Lo otro que resalta es que en ambos existe una preferencia a utilizar las estructuras escindidas en aquello que consideran la no izquierda, lo cual resulta lógico, dado que el recurso suele contrastar y significar a partir de la oposición.

Tabla 2: Frecuencia de escindidas en izquierda vs. no izquierda.

\begin{tabular}{lrrr}
\hline Tema & Anarquista & Socialdemócrata & Subtotal \\
\hline Izquierda & 8 & 1 & 9 \\
No izquierda & 10 & 5 & 15 \\
\hline Subtotal & 18 & 6 & 24 \\
\hline
\end{tabular}

Tanto la entrevista del anarquista como la del socialdemócrata comienzan con una definición y caracterización de la izquierda relativamente general, sin indicar un otro específico. Funcionan para preparar el escenario, a la vez que marcan las pautas sobre las que se genera la polémica y las alternativas dentro de 
las que se posiciona cada uno de los enunciadores. En el caso del anarquista, cuyo discurso es más dicotómico, esta definición la enuncia con construcciones escindidas.

(11) lo primero que tendríamos que decir es que en términos generales en una primera acepción muy amplia [. . .] la izquierda sería todos aquellos que han apostado por la transformación, por el progreso social, por el avance, por la reivindicación de las luchas históricas [...] y en ese sentido no hay una izquierda [...] (A5)

(12) En todo caso, a mí lo que me interesaría mucho plantear es en dónde se coloca la delimitación entre las distintas colindancias fronterizas entre lo que verdaderamente puede ser considerado de izquierda en un sentido genuino [.. .] frente a otras autodenominadas izquierdas. (A10)

Por la escindida en (11) se infiere que hay otros asuntos que no tendrían que plantearse primero para definir a la izquierda. Sin embargo, en este caso no es posible recuperar aquellas alternativas, pues podrían ser toda una serie de opciones con las que podría haber comenzado su respuesta. En ese sentido, la estructura pareciera funcionar como un marcador discursivo; no obstante, contrasta la alternativa explícita como la base de la definición, y es sobre ella que realiza precisiones en el transcurso de la entrevista. Por otra parte, (12) no sólo se presenta como la primera precisión de la definición general de (11), sino que la anula como la más interesante a su parecer, pues la escindida de (12), junto con el operador "en todo caso", implica que hay algo que no le interesa plantear: una acepción general, frente al constituyente escindido que resalta las fronteras de la izquierda y lo que queda fuera.

Por el contrario, la primera intervención sobre la izquierda que aparece en el discurso $\mathrm{S}$ no se expresa con oraciones escindidas, pero la mencionamos con el fin de comparar los acercamientos iniciales al tema por parte de ambos. El hecho de que en este discurso no se empleen estructuras escindidas en esta secuencia quizá pueda deberse a que sus fronteras son menos estrictas:

(13) el debate sobre la izquierda y su carácter se ha complicado últimamente, porque las novedades que han ocurrido [...] en las últimas décadas han esfumado delimitaciones, fronteras que estaban claramente delimitadas. [...] Creo que el arco de la izquierda en este momento va desde lo que podemos llamar posiciones claramente socialdemócratas, pero que aún entran en el campo de la izquierda, por un lado, hasta posiciones que podemos denominar de enfoques radicales revolucionarios. (S1-8) 
Decíamos que el discurso A es más dicotómico que éste, pues mientras en el primero se presentan dos polos tajantes: "lo que verdaderamente puede ser considerado de izquierda en un sentido genuino [. . . ] frente a otras autodenominadas izquierdas", en el segundo se trata de fronteras que, si bien tienen límites, no son claros; no se ponen frente a frente dos posturas, sino que la metáfora es de un arco en el que caben muchas. Pese a ello, no significa que la RS del socialdemócrata carezca de polos, sólo que en este ejemplo su registro es menos dicotómico. La alternativa eliminada se anula mediante una construcción restrictiva con relativa especificativa (no escindida): "pero que aún entran en el campo de la izquierda" implica, por un lado, que hay otras que no logran traspasar la delimitación y, por otro, que por lo general ninguna de ese tipo lo haría. De hecho, tal parece que para poder incluir a la que sí lo hace, es necesario indicar que se esfumaron delimitaciones.

Pocas líneas después, ambos hacen explícitas las orientaciones que se encuentran fuera del límite, a la vez que afirman su inclusión en la izquierda valiéndose del contraste. Los siguientes ejemplos son del discurso A:

(14) yo me ubicaría o aspiraría a ubicarme en [...] la izquierda radical, por lo menos aspiraría a ser radical, porque ¿quién expide los certificados de autenticidad? Pero lo cierto es que en un balance histórico en el caso incluso de la Ciudad de México, que se presenta como el epítome de la representatividad de gobiernos legítimos de izquierda [. . . ] dista mucho de serlo. (A18-21).

La secuencia (14) resulta interesante porque se vale de un tipo de estructura que, como se vio en la clasificación, no siempre es reconocida como escindida por no ser verbal. Sin embargo, resulta claro que genera un contraste. Por un lado, permite inferir que, frente a lo que sí es cierto (que los gobiernos del PRD, que han estado a cargo de la Ciudad de México, distan de la izquierda), hay algo que no es cierto (que alguien expida certificados de autenticidad). Profundizando, podríamos decir que, sin necesidad de certificados, el entrevistado es auténticamente de izquierda y que los gobiernos del PRD no lo son. La construcción expresa un procedimiento de desacreditación: mientras que para la radicalidad no es necesaria una valoración externa que certifique su autenticidad; para los gobiernos de la Ciudad de México que se presentan a sí mismos como de izquierda no existe duda de su descrédito.

Ahora bien, al analizar el discurso S notaremos también el uso del contraste para delimitar a la no izquierda de la izquierda:

(15) Quedan fuera a mi juicio de este arco de la izquierda posiciones socialdemócratas que podemos denominar reformistas en la tradición 
histórica de la socialdemocracia que en varios países de América Latina tiene impresiones muy claras. [...] Me refiero [...] a las tendencias que se vinculan con el Partido de la Revolución Democrática [PRD] y otras organizaciones menores, el PT [Partido del Trabajo], Movimiento Ciudadano [...] A mi juicio ahí está la frontera. Es decir, eso es lo que no entraría en el campo de la izquierda. A partir de ahí tenemos posiciones socialdemócratas pero avanzadas. (S10-15)

A diferencia de la secuencia referida antes (13), donde indicaba que las novedades esfumaron delimitaciones (para incluir a cierto tipo de socialdemocracia con la que se identifica), en este fragmento señala claramente lo que queda fuera: la socialdemocracia que él designa como reformista. Por otra parte, el contraste pragmático de la escindida implica que hay una alternativa eliminada con la que genera el contraste, algo que sí entraría en el campo de la izquierda: las posiciones socialdemócratas pero avanzadas, que se refieren a Morena. La necesidad de polarizar entre PRD y Morena, así como el uso de la restricción "pero", lleva a inferir que puede haber otros discursos pertenecientes a la polémica pública que considerarían que la socialdemocracia no suele ser avanzada, o que ambos partidos son parte de "lo que no entraría en el campo de la izquierda”. Efectivamente, para la postura anarquista es así. Como el mismo discurso S expresa, su grupo de pertenencia se ubica en la frontera última de las delimitaciones que marca ("a partir de ahí"), por lo que en el transcurso de la entrevista insiste en diferenciarse de las tendencias reformistas.

En cualquier caso, podemos afirmar que existen puntos de acuerdo entre ambos discursos, pues para los dos el PRD y otras organizaciones similares quedan fuera de la izquierda.

\subsection{Banderas de la izquierda: reforma o revolución}

Como se observa en la Figura 3, reforma y revolución constituye la segunda dicotomía más frecuente (22). En el discurso $\mathrm{S}$ se emplean más construcciones escindidas (13 frente a 9 del discurso A) en este rubro; de hecho, si se mira la Figura 2 que ilustra la RS de la socialdemocracia, se notará que el núcleo central está constituido por reforma, a la que divide en dos polos.

Es necesario precisar que cada uno entiende algo relativamente distinto por reforma y por revolución, así como por la relación entre ambas y los elementos asociados a ellas. No obstante, esto es parte de la polémica, y las dicotomías que refieren las estructuras escindidas tienen paralelos en ambos discursos. La principal diferencia reside en que en el discurso A la oposición principal se sostiene 
a nivel radical (de raíz), entre aspirar al poder (lo que relaciona con la reforma) y rechazar el poder (lo que relaciona con la revolución). Por su parte, en el discurso S no existe la segunda opción; incluso nunca es mencionada como una posibilidad que deba ser rechazada o incluida; la base es el uso del poder, y con él se puede aspirar a la revolución o a la reforma. Por ello, la polémica en $\mathrm{S}$ se da en un nivel que podríamos considerar de segundo orden.

(16) En su mayoría, la izquierda latinoamericana hoy, incluso la americana, se inclina por posicionamientos en el segundo campo, el de la búsqueda de esta construcción de mayorías que podrían operar en el campo electoral para definir quién controla los aparatos del Estado [...]. Y éste es un cambio que me parece que es el que caracteriza a la izquierda en las últimas dos décadas por lo menos [.. .] (S22-23)

El razonamiento que opera en (16) se vale del argumento de cantidad para desacreditar otras corrientes que no operan por la vía electoral. La construcción escindida que aparece en esta secuencia genera la inferencia de que existen otros cambios que no caracterizan a la izquierda. La alternativa eliminada con la que contrasta es la vía revolucionaria, como observamos en (17), donde explicita los posicionamientos que a su parecer no tienen éxito en la actualidad. Nótese, sin embargo, que no se trata de un procedimiento de exclusión con estos grupos, pues no los expulsa del ámbito de la izquierda, sino que simplemente les resta importancia y desacredita su alcance, al igual que en la siguiente secuencia:

(17) Si uno examina el "mercado" de ofertas políticas [.. .] de izquierda, encuentra muy pocos grupos que están ofreciendo la vía revolucionaria, la armada, de la toma de poder [...] No es que no existan, sino que no son los sectores que atraen a mayor número de miembros de la sociedad. (S 26-27)

La primera construcción escindida de (17) implica que no es relevante si existen o no los grupos radicales, sino que son irrelevantes por que no atraen a la sociedad general. Nuevamente se identifica el descrédito como un recurso típico del discurso polémico en $\mathrm{S}$; es decir, a la vez que disminuye la importancia de otros sectores, aumenta la del propio. Ello se puede derivar de la segunda estructura escindida de (17), la cual conduce a inferir que hay otros sectores que sí atraen a la sociedad: los grupos que buscan la construcción de mayorías electorales.

Cabe decir que, en la circulación global de discursos sobre el tema, son muchos los que respaldan este punto de vista al respecto de las opciones en la 
izquierda mexicana, con base en la irrupción de gobiernos de corte progresista que caracterizó los albores del siglo XXI en América Latina, los cuales opacaron las tendencias más radicales (cf. entrevistas a Meyer, Aboites, Semo y Modonessi en Centro de Documentación y Difusión de Filosofía Crítica 2013).

Los ejemplos vistos hasta este momento se enfocan en actores y sujetos políticos, aunque de manera abstracta; no obstante, más avanzada la entrevista, la polémica entre reforma y revolución deriva a un nivel teórico que remite a una formación discursiva socialista, como se advierte en los siguientes ejemplos:

(18) lo que decía [Rosa Luxemburgo] no era que la reforma había que excluirla de una perspectiva de izquierda revolucionaria. (S37)

(19) sino que quien hacía esa exclusión era el reformismo socialdemócrata. (S38)

(20) por tanto, lo que estaba afirmando es que la revolución debería reivindicar de manera adecuada la reforma como parte de su construcción revolucionaria. (S39)

Mediante las oposiciones presentadas en los fragmentos anteriores, el enunciador no sólo desdibuja las distinciones entre los planteamientos de reforma y revolución, sino que también crea una polarización entre dos tipos de socialdemocracia: la que llama avanzada o incluso radical (S52) y la que nombra aquí como reformismo socialdemócrata. Las alternativas eliminadas en la escindida de (18) se encuentran explícitas en (19) y (20). Nos detendremos en (19), la cual implica que hay alguien que no hace la exclusión de la reforma dentro de la perspectiva revolucionaria; tal alternativa se refiere a su grupo, el cual aboga por una tercera opción. Frente al reformismo socialdemócrata, hay una socialdemocracia que busca reformas "que conduzcan a cambios de carácter revolucionario” (S33). Expresión de lo anterior, de acuerdo con el enunciador, es el movimiento lopezobradorista (Morena):

(21) lo destacable en la izquierda mexicana ha sido la adopción de una línea política que [...] se ha centrado en la búsqueda de logros políticos por la vía electoral. (S133)

(22) Podemos rechazar en términos teóricos y políticos esta vía, puede gustarnos o no, lo que estoy señalando es que la realidad es que esto es lo que ha ocurrido. [...] Es el caso, por poner un ejemplo, del movimiento lopezobradorista 
que ha concluido en el último proceso electoral incluso con la elaboración de un proyecto alternativo de nación [...] que dibuja con toda claridad qué cambios se quieren hacer. (S134-135, 139)

De forma similar al empleo del argumento de cantidad con respecto a la vía electoral en América Latina (en contraposición a otros grupos minoritarios), en el caso de (21) se utiliza un argumento de superioridad, en donde el contraste con el resto de las alternativas las vuelve inferiores. Nuevamente, el modo de razonamiento polémico es el descrédito, más que la polarización. En otras palabras, la escindida lleva a inferir que hay otras líneas políticas que no son destacables, las cuales, recurriendo a segmentos anteriores, se refieren a las vías radicales revolucionarias. Cabe decir que la escindida en (21) es una construcción atípica dentro de la tipología, pero que cumple con la misma función pragmática y comparte características con las tradicionales. ${ }^{5}$

Por otro lado, las tres construcciones escindidas de (22) llaman la atención por encontrarse anidadas: (lo que estoy señalando es (que la realidad es (que esto es (lo que ha ocurrido)))). Identificamos esta estructura como una necesidad de defender el núcleo central de la representación (la reforma con perspectiva revolucionaria), al calificar e hipersubrayar un hecho como objetivo y real, frente a opiniones o preferencias que saldrían sobrando. De esta manera, la izquierda que busca la vía electoral, ejemplificada en Morena, resulta ser la que destaca en los hechos objetivos.

Ahora bien, en el discurso del anarquismo, que pareciera dialogar con lo anterior, no resultan relevantes los hechos calificados como objetivos; en contraste, el argumento que guía su discurso no es el de la cantidad ni la objetividad, sino la calidad y la autenticidad. El modo de razonamiento polémico que se expresa en (23) es el descrédito hacia la vía electoral como opción genuina y capaz de responder a las contradicciones sociales actuales y, aún más, la expulsión del campo de la izquierda de los grupos que ocupan puestos de elección:

(23) ¿Ésos son gobiernos de izquierda? Yo digo: bajo ninguna circunstancia. En todo caso a mí me parece que la pregunta que se tendría que plantear [...]

\footnotetext{
5 Carece de una oración relativa dentro de lo que sería la oración escindida, pues en este caso es no verbal y tampoco tiene una oración sustantiva comenzada por "que" en el constituyente escindido; es decir, no resulta el "es que" que une una parte con la otra y que en la literatura señalada y en el resto de los ejemplos de esta clase analizados sí tenía. Comparte el carácter especificativo y la cópula, y puede deshacerse eliminando el verbo ser, aunque con mayores transformaciones: "Destaca en la izquierda mexicana la adopción de una línea ..."
} 
es cuáles deberian ser las banderas de la izquierda [...]: ¿Aspirar al poder o luchar contra toda forma de poder que cristalicen gobiernos heterónomos fabricantes de subalternidad? Porque [...] las izquierdas genuinas del país ni aspiran ni buscan puestos de elección popular. (A28-30)

La escindida de (23) no sólo indica que la verdadera cuestión está en cuáles deben ser las banderas de la izquierda (esto es, el foco contrastivo), sino que no es relevante plantear si gobiernos como el del PRD en la Ciudad de México son de izquierda (A26), dado que la vía electoral no debería de ser una bandera. Esta operación refuerza la dicotomía entre reforma y revolución que, en el discurso A, radica no en el uso que se dé al poder, sino en el poder mismo y su necesaria ruptura para producir un cambio real.

En este contexto, donde lo esencial es ofrecer alternativas genuinas para superar al sistema, el discurso A desacredita explícitamente a Andrés Manuel López Obrador y su partido Morena:

(24) Los más de 15 millones de electores en el 2013 que votaron por López Obrador lo hicieron buscando una alternativa y lo que Andrés Manuel López Obrador les ha obsequiado es un alebrije; es decir otro partido más de la insulsa partidocracia. (A40-41)

La escindida en (24) crea un contraste pragmático entre lo que hizo (obsequiar un alebrije) y, por inferencia, lo que no hizo; es decir, crear una alternativa al poder para responder a las necesidades populares. Esta polémica circuló en su momento en el disenso público y generó expectativas más radicales, frente a los fracasos electorales, que nunca se concretaron y por las que López Obrador fue criticado (cf. entrevistas a del Valle y Fazio en Centro de Documentación y Difusión de Filosofía Crítica 2013). ${ }^{6}$ En este sentido, ¿cuáles son para el anarquista las alternativas que ofrece la izquierda radical para transformar la realidad lejos de las estructuras de poder? En principio: la ruptura del régimen, la revolución.

(25) ¿cuál es la alternativa para un cambio genuino en el país, la transición, vía acumulativa de reformas graduales, o la ruptura? Yo creo que en este país la ruptura se está haciendo cada vez más necesaria. El problema es que en

6 Véase al respecto la opinión de del Valle: "Cuando no gana, decide contener la movilización social. Él sigue sosteniendo que estuvo muy bien, porque entonces hubiera habido un enfrentamiento y un pueblo ensangrentado. El pueblo también es sabio y a veces las transformaciones reales y verdaderas implican violencia”. 
el país las contradicciones económico, políticas y sociales no han sido acompasadas por un desarrollo análogo [.. .] de la conciencia política revolucionaria capaz de cristalizar en nuevas construcciones frente a la bancarrota rotunda de esas formas heterónomas de organización que son los partidos políticos. (A140-142)

Si bien en el discurso S el argumento está sobre la cantidad vista como una realidad; en el discurso A se encuentra sobre otra clase de hechos, como la necesidad de la ruptura frente a contradicciones irreparables, $y$, gracias a la escindida en (25), se infiere que ello no representa un problema; en este sentido, polemiza con los discursos contrarios, como el de S, que circulan en el disenso público y que califican de innecesaria la ruptura. A pesar de que admite como una dificultad la falta de desarrollo de la conciencia revolucionaria requerida para acompañar la transición, más adelante presenta soluciones y ejemplos de organizaciones que las han llevado a cabo:

(26) lo que tenemos que hacer es [...] entender en mucho el ejemplo que desde las perspectivas anarquistas, sobre todo en México, se están dando para reflotar, pues para mí los tres referentes que son los desafios programáticos que la izquierda revolucionaria tendría que visualizar para hacer tierra [...] son indudablemente autonomía, autogobierno y autogestión. [...] (A202)

(27) [...] por eso es que reivindico tanto al zapatismo, por eso soy subscriptor de la sexta declaración de la selva lacandona (A205)

Las dos construcciones escindidas en (26) marcan, por un lado, los medios para construir soluciones y alternativas: la autonomía, el autogobierno y la autogestión, cuyo ejemplo lo dan las perspectivas anarquistas y el zapatismo, y por otro, generan un contraste con las opciones eliminadas pragmáticamente. Estas opciones no explícitas en el discurso aledaño, relacionadas a la vía de la reforma con la que establece una polarización tajante, pueden encontrarse más adelante en la entrevista: "en contra de todos los principios de autoridad y todas las formas que reproduzcan poderes heterónomos que impiden que los grupos [.. .] tomen en sus manos la resolución de los asuntos que directamente [...] les afectan e interesan, que finalmente ése es un elemento que sintetiza este tríptico virtuoso que es la autonomía, el autogobierno y la autogestión. Curiosamente todas las izquierdas convencionales a estas tres categorías le huyen como la peste” (263-265).

Así pues, mientras para el discurso A la polarización es entre la vía de la reforma y la de la revolución de forma tajante; para el discurso S la polarización es entre una reforma de alcances reformistas y una reforma con perspectivas 
revolucionarias, a la vez que desacredita la fuerza y las posibilidades de la vía radical revolucionaria.

\subsection{Alcances de la izquierda: anticapitalismo vs. antineoliberalismo}

Finalmente, existe otra dicotomía sobre la que polemizan las dos corrientes aquí discutidas, la cual opera en un nivel más sutil, pero que está intrínsecamente relacionada con los alcances de la transformación y con las vías para conseguirla. Se trata del adversario y los fines últimos y posibles, de aquello contra lo que lucha la izquierda para lograr la igualdad. En los entrevistados, los elementos de esta dicotomía adquieren tintes axiológicos y deónticos: para ser de izquierda se necesita ser anticapitalista o, por lo menos, antineoliberal; no es de sorprender, dado que el anticapitalismo es uno de los pilares históricos de la izquierda, como herencia del socialismo, y su mayor crítica es hacia las diferencias de oportunidades que genera este sistema en la sociedad (Stolowicz 2007). No obstante, desde finales del siglo XX, con el crecimiento del neoliberalismo y la irrupción de movimientos altermundistas y gobiernos de corte progresista posteriores, el foco de ataque comenzó a cambiar de lugar (Huerta 2011). Tal como lo menciona el entrevistado socialdemócrata: "estas dos categorías de antineoliberal y anticapitalista están definiendo los campos de delimitación de la izquierda en los últimos tiempos." (S 27) En la secuencia siguiente observamos el punto de vista en el discurso S:

(28) El último punto que quería señalar es que hay un campo en el que también se intenta delimitar lo que es izquierda de lo que no lo es. Evidentemente los ejemplos que he señalado se colocan en una izquierda que podemos denominar izquierda antineoliberal. Esta izquierda que se manifiesta como antineoliberal es la izquierda predominante en América Latina... (S 53-54)

Las estructuras escindidas en (28) subrayan la importancia de la distinción para la izquierda. En la primera funciona casi como un marcador discursivo, similar a la construcción estudiada en (11), para delimitar el último asunto relevante dentro de su definición, frente a cualquier otro que podría haber ocupado su lugar. La segunda estructura recurre nuevamente al argumento de cantidad empleado para resaltar el valor de su grupo y desacreditar el de otros; la alternativa inferida pragmáticamente es, nuevamente, la de la vía revolucionaria, la cual asocia con el anticapitalismo, como se advierte en otra parte de la 
entrevista: "[Hay] otros que [...] proponen cambios revolucionarios anticapitalistas, pero que no definen con claridad o por lo menos no resultan claros para la mayoría de los ciudadanos cuáles son los medios que plantean para lograr esos fines o plantean medios que no son aceptados por la mayoría." (S18-19).

Además, conviene recordar que el socialdemócrata se diferencia, creando una polarización, del grupo que llama "socialdemocracia neoliberal" (S142), la cual sí expulsa del ámbito de la izquierda por alinearse a dicho sistema económico.

El debate radica, entonces, entre un cambio de sistema (anticapitalismo) y un cambio de modelo económico (antineoliberalismo) (Huerta 2011). Es decir, existe una polémica pública entre las dos formas de concebir los fines últimos y los inmediatos, de manera que el antineoliberalismo es más propio de la socialdemocracia avanzada, desde la perspectiva de $\mathrm{S}$, y el anticapitalismo, de las vertientes radicales. $\mathrm{Al}$ respecto, el discurso $\mathrm{A}$, menciona:

(29) la izquierda ha sido muy importante en contribuir al agotamiento del ciclo vital del capitalismo salvaje [...] Aunque muchas otras expresiones desde cierta izquierda lo que están planteando es la vuelta nostálgica al estado interventor de corte keynesiano, que es ahí donde yo ya no reconozco la diferencia entre la izquierda y la derecha. (A30-31)

Es decir, la primera escindida en (29) polariza entre dos planteamientos: la vuelta a un estado interventor (que, podemos inferir, se refiere a toda izquierda que se valga de las estructuras de poder para otorgar beneficios a sus ciudadanos) y la alternativa eliminada por contraste: contribuir a agotar el capitalismo. Es decir, ese tipo de izquierda no sólo plantea un estado interventor, sino que no contribuye a abolir el capitalismo. La polarización es tan acentuada que funciona para calificar a estas corrientes como de derecha, tal como se subraya mediante la segunda escindida. Entre todas las razones por las que haría esa afirmación, el hecho de no sumarse a terminar con el capitalismo lo lleva a diferenciarlos de la izquierda.

Por otra parte, en el discurso A, también la distinción con el neoliberalismo (mas no especifica que deba ser anticapitalista) funciona como un argumento de calidad que asegura la autenticidad de la izquierda. En ese caso, la escindida (30) permite a inferir que hay otras izquierdas que no se diferencian del neoliberalismo y que, por tanto, no son genuinas:

(30) la izquierda genuina es aquella que se diferencia de los discursos privatistas que se resumen en el programa del neoliberalismo, en la privatización a ultranza de las cosas, el libre juego de las fuerzas del mercado (A36). 
No obstante lo visto en esta dicotomía, es cierto que la distinción entre anticapitalismo y antineoliberalismo no posee un fuerte efecto polarizante aún, pues su data histórica es relativamente reciente (de los ochenta para acá), de manera que la polémica a la que da lugar aún tiene camino por recorrer (Huerta 2011). Hasta ahora, en realidad, ambos en algún momento se asumen a sí mismos, o a la izquierda en la que se insertan ideológicamente, como anticapitalistas y/o antineoliberales (A36). En este sentido, desde la perspectiva de S, las tendencias latinoamericanas que se diferencian del neoliberalismo son también anticapitalistas: "Y claramente esta tendencia antineoliberal de la izquierda se autodefine de manera directa como anticapitalista” (S56). La distinción tajante sigue siendo la clásica: entre capitalistas y opositores al capitalismo y a sus derivaciones económicas o culturales (es decir, socialistas, anarquistas, comunistas, etcétera). Por esta razón, tanto en la RS del anarquismo como en la de la socialdemocracia (Figuras 1 y 2), se sitúa el elemento "neoliberalismo y capitalismo" en el polo negativo, pero no hay una distinción entre antineoliberales por un lado y anticapitalistas por otro. De hecho, estar contra el neoliberalismo es uno de los aspectos que estas dos corrientes tan distantes tienen en común, por lo que puede ser uno de los puntos de acuerdo en la palestra pública, dentro de la cual la polémica gestiona el conflicto.

\section{Conclusiones}

En este capítulo se ha observado que la polémica entre el anarquismo y la socialdemocracia se basa en una toma de posición del enunciador dentro de una serie de alternativas conflictivas que expresan diversos puntos de vista, entre los cuales se contrasta uno y se anula otro $u$ otros. En esta interacción no directa, en la que está en disputa el derecho a definir e identificarse con la izquierda, el modo de razonamiento en el discurso del anarquismo es principalmente la polarización entre un nosotros frente a un ellos (la socialdemocracia) que excluye, pero que también desacredita al posicionar a ambos bandos en los extremos de las diferentes dicotomías analizadas aquí, sin apenas distinguir matices dentro del ellos. Por su parte, el discurso de la socialdemocracia emplea sobre todo la desacreditación con respecto a las perspectivas radicales, a las que resta importancia y nulifica, pues incluso no contempla la posibilidad de que no empleen el poder, como reivindica el discurso A; en cambio, las mayores polarizaciones las hace para oponer dos posiciones que en el debate general pueden presentarse sin matices: una socialdemocracia reformista y otra con alcances revolucionarios. 
Si bien las grietas en la polémica parecen infranqueables entre estas dos izquierdas, es cierto que ambas comparten un mismo espacio caracterizado por la búsqueda de alternativas para la construcción de un futuro donde las desigualdades sociales sean cada vez menores. La posibilidad de gestionar el conflicto, algo que ofrece la polémica, es esencial para ir conformando la viabilidad de cualquier proyecto.

Ahora bien, el potencial de análisis que ofrecen las construcciones escindidas resulta muy productivo en el discurso político, dado que permite observar en un nivel sintáctico pragmático no sólo la construcción del sentido de un enunciado por medio de inferencias, que pueden o no encontrarse en otras secuencias del discurso, sino también la puesta en escena de voces que se confrontan para actualizar el sentido en una dimensión polémica, dentro de la cual el locutor se posiciona. Funcionan como un recurso para incorporar la "palabra ajena" (Bajtín 1976 es decir, como indicadores de polifonía (al igual que otras formulaciones sintácticas más trabajadas como la paráfrasis, el discurso referido, o bien, el uso de opciones léxicas, procedimientos de cita, entre otros), el cual posee un potencial polémico ligado al contraste pragmático que genera.

Sin duda, hay asignaturas pendientes; el estudio de más corpus orales o escritos todavía tiene mucho que ofrecer, por ejemplo, para poder afirmar que existe una relación entre el uso de ciertas construcciones sintácticas y ciertos géneros discursivos o medios, como ya se ha hecho en relación con el léxico o vocabulario perteneciente a determinados campos. Por ahora, sólo podemos confirmar la importancia de incorporar una mirada sintáctico-pragmática en el abordaje del análisis del discurso con el fin de identificar y explicar la construcción de sentidos en determinado discurso puesto en relación con los otros cuyo espacio comparte en la sociedad.

\section{Referencias}

Abric, Jean-Claude. 2004. Prácticas y representaciones sociales. México: Ediciones Coyoacán. Amossy, Ruth. 2017. Apología de la polémica. Buenos Aires: Prometeo.

Atayan, Vahram y Wienen, Ursula. 2014. Inferential cleft constructions in translation. En AnnaMaría De Cesare (ed.). Frequency, Forms and Functions of Cleft Constructions in Romance and Germanic: Contrastive, Corpus-Based Studies, 345-376. Berlin/Boston: De Gruyter. Authier-Revuz, Jacqueline. 1995. Ces mots qui ne vont pas de soi. Boucles réflexives et noncoïncidences du dire. Paris: Larousse.

Bajtín, Mijaíl. 1979. Estética de la creación verbal. México: Siglo XXI.

Bingono, Emmanuel. 2011. La théorie du noyau central: entre continuité des représentations collectives et de la spécificité de la psychologie sociale. La recherche en education 6. 21-38. 
Bobbio, Norberto. 1995. Derecha e izquierda. Significado de una distinción política. Madrid: Taurus.

Bosque, Ignacio y Javier Gutiérrez-Rexach. 2009. Fundamentos de sintaxis formal. Madrid: AKAL.

Bublitz, Wolfram. 2011. Cohesion and coherence. En Jan Zienkowski, Jan-Ola Östman y Jef Verschueren (eds.). Discursive pragmatics. Handbook of Pragmatics Highlights, vol. 8, 37-49. Amsterdam: John Benjamins.

Calude, Andreea. 2009. Cleft Constructions in Spoken English. Saarbrüchen: Verlag Dr. Müller.

Centro de Documentación y Difusión de Filosofía Crítica. 2013. Izquierdas mexicanas en el siglo XXI. México: Universidad Nacional Autónoma de México, Centro de Investigaciones Interdisciplinarias en Ciencias y Humanidades. En línea https://www.youtube.com/chan nel/UCreljr_Mq9IEe-_3pz_NQ3Q.

Charaudeau, Patrick. 2001. De la competencia social de la comunicación a las competencias discursivas. Revista Latinoamericana de Estudios del Discurso 1(1). 7-22.

Collins, Peter. 1991. Cleft and Pseudo-cleft constructions in English. London/New York: Routledge.

Cortés, Luis y Raquel Hidalgo. 2015. Indicadores de cambio temático en el discurso parlamentario: Análisis de un discurso de Rodríguez Zapatero [2011]. Revista Signos. Estudios de Lingüística 48(89). 279-306.

De Cesare, Anna-Maria. 2014. Cleft constructions in a contrastive perspective. Towards an operational taxonomy. En Anna-María De Cesare (ed.). Frequency, Forms and Functions of Cleft Constructions in Romance and Germanic: Contrastive, Corpus-Based Studies, 9-48. Berlin/Boston: De Gruyter.

De Cesare, Anna-Maria, Davide Garassino, Rocío Agar Marco y Laura Baranzini. 2014. Form and frequency of Italian Cleft constructions in a corpus of electronic news. A contrastive perspective with French, Spanish, German and English. En Anna-María De Cesare (ed.). Frequency, Forms and Functions of Cleft Constructions in Romance and Germanic: Contrastive, Corpus-Based Studies, 49-99. Berlin/Boston: De Gruyter.

Ducrot, Oswald. 1984. Le dire et le dit. Paris: Minuit.

Erdmann, Peter. 1990. Fokuskonstruktioinen im Deutschen und Englischen. En Claus Gnutzmann (ed.). Kontrastive Linguistik, 69-83. Bern: Peter Lang.

España Villasante, Margarita. 1996. Aspectos semántico-pragmáticos de la construcción es que en español. DICENDA. Cuadernos de Filología Hispánica 14. 129-147.

Fernández Leborans, María Jesús. 1992. La oración del tipo es que ... Verba 19. 223-239. Garand, Dominique. 1989. La griffe du polémique. Montréal: L’Hexagone.

Garassino, Davide. 2014. Cleft sentences. Italian-English in contrast. En Anna-María De Cesare (ed.). Frequency, Forms and Functions of Cleft Constructions in Romance and Germanic: Contrastive, Corpus-Based Studies, 101-138. Berlin/Boston: De Gruyter.

García Negroni, María Marta. 2009. Dialogismo y polifonía enunciativa. Apuntes para una reelaboración de la distinción discurso/historia. Palabras de guarda 7. 11-27.

Gutiérrez Bravo, Rodrigo. 2008. La identificación de los tópicos y los focos. Nueva Revista de Filología Hispánica 56(2). 363-401.

Gutiérrez Ordoñez, Salvador. 2015. La familia de las ecuacionales. Revista Internacional de Lingüística Iberoamericana 2(26). 15-37.

Huber, Stefan. 2006. The complex function of it-clefts. En Valéria Molnár y Susanne Winkler (eds.). The Architecture of Focus. 549-578. Berlin/New York: De Gruyter. 
Huerta, Enrique. 2011. Antineoliberalismo y anticapitalismo en América Latina: el "modelo económico", la respuesta social y sus perspectivas políticas. Tesis de licenciatura. Universidad Nacional Autónoma de México.

Kiss, Katalin. 1998. Indentificational focus versus informational focus. Language 74(2). 245273.

Lambrecht, Knud. 2001. A framework for the analysis of cleft constructions. Linguistics 39. 463-561.

Maingueneau, Dominique. 2003. Los términos clave del análisis del discurso. Buenos Aires: Nueva Visión.

Montolío, Estrella, Mar Garachana y Marisa Santiago Barriendos. 2001. Manual de escritura académica. Barcelona: Ariel.

Moscovici, Serge. 1988. Notes towards a description of social representations. European Journal of Social Psychology 18. 211-250.

Murguía, Nélida. 2016. Focalización y modalidad en la construcción discursiva de tres movimientos de izquierda: lenguaje y representaciones sociales. Tesis de maestría. Universidad Nacional Autónoma de México.

Nascimento, Erivardo. 2015. A polifonia nos gêneros acadêmicos e formulaicos: a construção de sentidos a partir da evocação da palavra alheia. Letras de Hoje 50(3). 342-351.

Real Academia Española y Asociación de Academias de la Lengua Española. 2010. Nueva Gramática de la Lengua Española. Manual. Madrid: Espasa Calpe.

Smead, Robert. 1994. En torno al modo en oraciones seudo-escindidas y exclamativas: patrones entre monolingües y bilingües. Hispania 77(4). 842-853.

Stolowicz, Beatriz. 2007. Gobiernos de izquierda en América Latina. Un balance político. Bogotá: Aurora.

Van der Wal, Jenneke. 2015. Information structure, (inter)subjetivity and objetification. Journal of Linguistics 51(2). 425-464.

Woolf, Virginia. 2013. La señora Dalloway. Buenos Aires: Debolsillo. 